\title{
Establishment of a lactation management centre at Teaching Hospital, Kandy and its impact on admissions to the special care baby unit
}

\author{
S C Wickramasinghe ${ }^{1}$, T M S M Dharmaratne ${ }^{2}$, W A C S Wickramasinghe ${ }^{3}$
}

Sri Lanka Journal of Child Health, 2014; 43: 159-162

\begin{abstract}
Background: A lactation management centre (LMC) was established in July 2006 at General Hospital, Kandy to handle problems with breast feeding in neonates and young infants. Prior to that, babies with feeding problems needed to be admitted to the special care baby unit (SCBU) even if the problem was a minor one needing few hours of attention.
\end{abstract}

Objectives: To compare the admissions to SCBU related to breast feeding before and after establishment of LMC and to determine if the LMC has contributed significantly to reduce the burden of admissions to SCBU.

Method: A retrospective analytical study was carried out analysing 2724 admissions spanning over a period of 4 years, 2 years before and 2 years after establishment of the LMC. Data was collected from the admission register of SCBU. Babies admitted from the postnatal wards with "Reason for admission" being clearly related to feeding problems were enrolled. The final diagnosis, as mentioned in the admission register, was essentially considered. Babies who had other concurrent pathologies, direct admissions from outpatient department and transfers from outstations were excluded. Each case record was personally scrutinized by the chief investigator.

Results: There were 1170 and 1554 admissions to SCBU from July 2004 to June 2006 and July 2006 to June 2008 respectively. Out of them 684 admissions were directly related to a breast feeding problem in the former 2 years and it has been reduced to 674 in the latter 2 years. Out of them the babies who stayed more than 72 hours in the unit were excluded and the analysis was done on 550 and 639 admissions respectively.

${ }^{1}$ Consultant Paediatrician, ${ }^{2}$ Medical Officer in Health Informatics, Health Information and Research Unit, ${ }^{3}$ House Officer in Paediatrics, General Hospital (Teaching) Kandy

(Received on 20 January 2014: Accepted after revision on 21 February 2014)
Conclusion: Admissions to SCBU related to breast feeding problems have significantly reduced $(\mathrm{P}=0.002)$ after the establishment of the LMC.

(Key words: Breast feeding; lactation management centre; neonates; mother baby unit; special care baby unit)

\section{Introduction}

Problems related to breast feeding are relatively common among mothers and newborn babies since it is a skill that both of them have to develop. Often these are overlooked by the staff in busy postnatal wards. After a normal delivery mother gets discharged within 24 hours. Though continuum of care is expected by the field health staff, feeding problems are not always correctly addressed. A small percentage of these mothers will come back to postnatal clinics or paediatric clinics, but the time spent on each patient by the staff is not adequate to deal with a problem in breast feeding. Opinion given by elders, relatives and friends play a major role, and with the ready availability of infant formula milk products, mothers opt for bottle feeding the baby, depriving many valuable benefits of breast milk to the young infant. If the feeding problem becomes severe enough, they need to be admitted to the Special Care Baby Unit (SCBU) which leads to many disadvantages like mother-baby separation, inability to do demand feeding, risk of cross infection, cost to the health sector etc.

The need to help mothers with problems in breast feeding has been long felt by many people involved in the field of maternal and newborn care worldwide but establishment of a "Lactation Management Centre" (LMC) for that purpose has been truly a Sri Lankan concept. The first LMC in the world was started in Castle Street Women's Hospital in Colombo, Sri Lanka in the year $2000^{1}$. Another LMC has been initiated in Rajshahi in India in $2004^{2}$. LMC can run as a separate day centre or be a part of a mother-baby unit (MBU) ${ }^{3}$. Latter has the advantage of admitting babies with minor problems for overnight observation. 
General Hospital, Kandy (GHK) boasts of the $2^{\text {nd }}$ LMC in Sri Lanka which was started on $06^{\text {th }}$ July 2006. It is under the supervision of the consultant paediatrician in-charge of SCBU. Medical cover is provided by medical officers in SCBU. Work is mainly managed by 4 nursing officers who are trained in breast feeding. These training programmes include a 5-day breast feeding counselling course and a 3-day baby friendly hospital initiative (BFHI), both funded and coordinated by the Family Health Bureau of Sri Lanka.

LMC at GHK functions as a day centre, $7 \mathrm{am}$ to $4 \mathrm{pm}$, 7 days a week. No admissions are made due to restricted space and limitation in other facilities. Nurses from LMC do daily visits to 3 postnatal wards, one nurse/ward basis. Feeding problems which need more time to resolve are brought to the LMC. Referrals are made through many sources viz. by the SCBU medical officers during their routine postnatal newborn checkups, from the Out Patients Department (OPD) or clinics, other wards like paediatric surgical unit, general paediatric unit, field health staff or self-referrals. There is also a hot line to get advice. Mothers who delivered at GHK are given this information in the form of a rubber stamp on baby's child health development record (CHDR).

LMC provides a relaxed environment where mothers can spend a few hours. In addition, they are trained on expression of breast milk, cup or spoon feeding if indicated. Back massage is given to mothers who have concern about the flow of milk. Accurate measurement of baby's weight is done using an electronic weighing scale. Subsequent follow up visits are arranged by the nurses. Any medical, surgical or obstetric problems identified at LMC are brought to the attention of the medical officers or the consultant paediatrician

\section{Objectives}

1. To compare the admissions to SCBU related to breast feeding before and after establishment of LMC
2. To determine if the LMC has contributed significantly to reduce the burden of admissions to SCBU.

\section{Method}

A retrospective analytical study was carried out analysing 2724 neonates admitted to SCBU from 01 . 07.2004 to 30.06 .2008 to cover a period of 4 years, 2 years before and 2 years after the establishment of the LMC. Data was collected from the admission register and each case record was individually scrutinized by the investigators.

Eligibility criteria comprised babies of both sexes and babies admitted from postnatal wards with problems related to breast feeding which was given as the cause of admission on admission register. These included dehydration fever, low random blood sugar, exaggerated physiological jaundice, poor sucking, delayed establishment of breast feeding and breast problems/flat nipples.

In each case the number of days in the SCBU and the final diagnosis as written on the admission register was taken into consideration. This helped us to exclude some of the cases which can overlap with other underlying pathologies.

Exclusion criteria comprised babies admitted from OPD/clinics, transfers from outstations, low birth weight babies less than $1.5 \mathrm{~kg}$, babies who needed to be in SCBU for more than 72 hours, .babies with incomplete information on the admission register, babies with any additional problems which may have contributed to poor feeding e.g. Down syndrome, and other documented causes which may have contributed to jaundice, if that was the reason for admission to SCBU e.g. Rh or ABO incompatibility.

Ethical approval was obtained from the Kandy Hospital Ethical Review Committee. Data was analyzed using a Chi-squared distribution table $\mathrm{e}^{4,5}$.

\section{Results}

Summary of the collected data is shown in Table 1.

Table 1: Summary of the collected data

\begin{tabular}{|l|c|c|c|c|}
\hline & July 2004 - June 2006 & \% & July 2006 - June 2008 & \% \\
\hline Total admissions to SCBU & 1170 & & 1554 & 43.4 \\
\hline Total admissions related to BF & 684 & 58.5 & 674 & 41.1 \\
\hline Total admissions $<72$ hrs. related to BF & 550 & 47.0 & 639 & 4 \\
\hline
\end{tabular}

$S C B U$ - Special care baby unit, BF-breast feeding 
Contingency table is shown in Table 2.

Table 2: Contingency table

\begin{tabular}{|c|l|c|c|c|}
\hline & & July 2004 - June 2006 & July 2006 - June 2008 & Total \\
\hline 1 & $<72$ hr admissions with BF problems & 550 & 639 & 1189 \\
\hline 2 & Other admissions & 620 & 915 & 1535 \\
\hline 3 & Total admissions & 1170 & 1554 & 2724 \\
\hline
\end{tabular}

BF-breast feeding

For analysing purpose Chi-square test of independence was used

$$
\begin{aligned}
\text { Chi-square }= & \frac{(a d-b c)^{2}(a+b+c+d)}{(a+b)(c+d)(b+d)(a+c)} \\
& 9.41
\end{aligned}
$$

$$
\text { Degree of freedom }=1 \quad \mathrm{P}=0.002
$$

According to Chi squared distribution table; Chisquare of 9.41 at degree of freedom 1 the probability is below 0.01 The noted difference in admissions in this study is highly significant at $1 \%$ level; hence LMC is efficacious in reducing number of admissions to SCBU. The probability of getting this type of difference in admissions after establishment of a well supervised, effectively functioning LMC is $99 \%$

\section{Discussion}

For the period from July 2004 to June 2006, 58.5\% of in-ward admissions were due to a problem directly related to breast feeding. Out of them some were excluded from the study due to more than 72 hours long stay in the unit. This percentage has dropped after the intervention to $43.4 \%$. According to the inclusion and exclusion criteria only $47 \%$ and $41.1 \%$ of the above figures were respectively considered for the study.

Establishment of an effectively functioning LMC in a health facility where the neonates and young infants are cared should definitely have a favourable influence on the burden of admissions to SCBU. Our study showed a significant reduction on the number of admissions to SCBU due to feeding problems following the establishment of LMC. We had to do stringent scrutinizing of data to minimize false inclusions and false exclusions.

Feeding problems become a part of many illnesses in neonates. At the same time it may be the first symptom of a serious underlying illness. Therefore, a prospective study with careful analysis of each and every newborn admitted, will give the most accurate results.

Having a mother-baby unit where overnight admissions and observation can be done will have the most significant contribution to reduce the admissions to SCBU. This will help with the postnatal ward overcrowding as well. Many of the Base and General hospitals in the country do have LMCs but the efficient functioning of these always depend on the driving force provided by the consultant paediatrician/neonatologist in charge and the hospital administration. Newborn baby has the right to receive his/her most valued gift by nature, the mother's milk. As health care staff we are bound for the commitment to facilitate this.

\section{Conclusion}

Admissions to SCBU related to breast feeding have significantly reduced $(\mathrm{P}=0.002)$ after the establishment of the LMC.

\section{Recommendations}

1. Multicentre study where LMC's have been established recently, since no studies have been done on this initiative so far.

2. Regular refresher courses for the LMC staff to maintain their efficiency.

\section{Acknowledgements}

We thank the staff of LMC and SCBU at GHK, present two consultants of SCBU Dr. (Mrs.) K. Epitakumbura and Dr. (Mrs.) Nalika Menike and staff of Medical record room, GHK.

\section{References}

1. Wickramasinghe SC. Lactation management centres: A step forward in successful breast 
feeding. Sri Lanka Journal of Child Health, 2012; 41(2): 82-90.

http://dx.doi.org/10.4038/sljch.v41i2.4397

2. Hossain MA, Haque MI, Siddiqui AB, Bari MI. Activities of Lactation Management Centre of Rajshahi. TAJ 2005; 18(2): 89-92.

3. Family Health Bureau, Ministry of Health Care and Nutrition; Perinatal Society of Sri Lanka; Central Engineering Consultancy Bureau. Building \& other guidelines for Neonatal Intensive Care Units, Special Care Baby Units, Mother Baby Centre; 2007.
4. Mahajan BK. Methods in Bio statistics for medical students \& research workers. $7^{\text {th }}$ ed. Khanal AB, editor. Jaypee (New Delhi); 2010.

5. Department of Mathematics and Computer Science [home page on the Internet]; Hobart and William Smith Colleges, Geneva. The Chi Square Statistics. [cited 2013 Oct 02] Available from:

http://math.hws.edu/javamath/ryan/ChiSquare.ht $\underline{\mathrm{ml}}$ 